\title{
Chronic dietary supplementation with turmeric protects against 1-methyl-4-phenyl-1,2,3,6-tetrahydropyridine-mediated neurotoxicity in vivo: implications for Parkinson's disease
}

\author{
Rajeswara Babu Mythri ${ }^{1}$, Jayagopalan Veena ${ }^{2}$, G. Harish ${ }^{1}$, B. S. Shankaranarayana Rao ${ }^{2}$ and \\ M. M. Srinivas Bharath ${ }^{1 *}$ \\ ${ }^{1}$ Department of Neurochemistry, National Institute of Mental Health and Neurosciences (NIMHANS), PB NO. 2900, \\ Hosur Road, Bangalore 560 029, Karnataka, India \\ ${ }^{2}$ Department of Neurophysiology, National Institute of Mental Health and Neurosciences, PB No. 2900, Hosur Road, \\ Bangalore 560 029, Karnataka, India
}

(Received 15 May 2010 - Revised 1 October 2010 - Accepted 14 December 2010 - First published online 8 April 2011)

\section{Abstract}

Multiple pathways including oxidative stress and mitochondrial damage are implicated in neurodegeneration during Parkinson's disease (PD). The current PD drugs provide only symptomatic relief and have limitations in terms of adverse effects and inability to prevent neurodegeneration. Therefore, there is a demand for novel compound(s)/products that could target multiple pathways and protect the dying midbrain dopaminergic neurons, with potential utility as adjunctive therapy along with conventional drugs. Turmeric is a spice used in traditional Indian cuisine and medicine with antioxidant, anti-inflammatory and potential neuroprotective properties. To explore the neuroprotective property of turmeric in PD, mice were subjected to dietary supplementation with aqueous suspensions of turmeric for 3 months, mimicking its chronic consumption and challenged in vivo with 1-methyl-4-phenyl-1,2,3,6-tetrahydropyridine (MPTP). Brain samples from untreated and treated groups were characterised based on mitochondrial complex I (CI) activity, protein nitration and tyrosine hydroxylase immunoreactivity. Chronic turmeric supplementation induced the enzyme activity of $\gamma$-glutamyl cysteine ligase, which in turn increased glutathione levels and protected against peroxynitrite-mediated inhibition of brain CI. These mice were also protected against MPTP-mediated protein nitration, CI inhibition and degeneration of substantia nigra neurons in the brain. We conclude that chronic dietary consumption of turmeric protects the brain against neurotoxic insults, with potential application in neurodegeneration. Further characterisation of the active constituents of turmeric that potentially promote neuroprotection could improve the utility of dietary turmeric in brain function and disease.

Key words: Turmeric: Parkinson's disease: 1-Methyl-4-phenyl-1,2,3,6-tetrahydropyridine: Protein nitration: Mitochondrial complex I

Parkinson's disease (PD) is a movement disorder, pathologically manifested by the loss of dopaminergic neurons in the substantia nigra (SN) of the midbrain $(\mathrm{MB})$ with concomitant dopamine depletion in the striatum $(\mathrm{St})^{(1)}$. Currently, there are several options for PD pharmacotherapy including Levodopa (L-DOPA), dopamine receptor agonists, monoamine oxidase-B inhibitors, etc. ${ }^{(2)}$. These therapeutic strategies are mainly symptomatic and strive to replenish striatal dopamine, but their ability to prevent or slow down neurodegeneration of $\mathrm{SN}$ neurons has not been validated in humans. Hence, novel compounds that prevent neurodegeneration with proven utility as adjunctive therapy in PD need to be explored.

The curry spice turmeric has been used in traditional Indian cuisine and medicine for centuries. Curcumin, the major polyphenol from turmeric, possesses diverse biological and medicinal properties with potential application in several diseases ${ }^{(3,4)}$. We demonstrated that curcumin protects against peroxynitrite (PN)-mediated protein nitration and mitochondrial dysfunction $^{(5)}$ and against glutathione (GSH) depletion-mediated oxidative damage ${ }^{(6,7)}$. Curcumin is neuroprotective in various

\footnotetext{
Abbreviations: b.w., body weight; CI, mitochondrial complex I; $\gamma$-GCL, $\gamma$-glutamyl cysteine ligase; GSH, glutathione; MB, midbrain; MPTP, 1-methyl-4phenyl-1,2,3,6-tetrahydropyridine; PD, Parkinson's disease; PN, peroxynitrite; SN, substantia nigra; St, striatum; TH, tyrosine hydroxylase.

*Corresponding author: Professor M. M. Srinivas Bharath, fax +91 8026564830 , email bharath@nimhans.kar.nic.in

†Present address: Laboratoire Psynugen, UMR INRA 1286, CNRS 5226, Université Bordeaux 2, Bâtiment UFR Pharmacie, 2ème Tranche, 2ème Etage, CC34, Université Victor Segalen Bordeaux 2, 146 rue Léo Saignat, 33076 Bordeaux Cedex, France.
} 
models of neurodegeneration, emphasising its therapeutic potential in $\mathrm{PD}^{(8)}$.

Curcumin in the traditional sense is considered to be more of a dietary agent than a drug. Consequently, the clinical application of curcumin at higher doses has faced certain limitations. These include poor bioavailability ${ }^{(9-11)}$ and toxicity $^{(12)}$. Curcumin exposure induced the expression of leucine-rich repeat kinase 2 protein, which is part of neurotoxic inclusions found in neurodegenerative disorders ${ }^{(13)}$. Curcumin exacerbated paraquat-mediated neurotoxicity in dopaminergic neurons ${ }^{(14)}$, indicating that the medicinal benefits of curcumin in isolation need to be re-evaluated. Chronic consumption of curcumin as turmeric in diet throughout life could be more effective for optimum neuroprotective effect in the brain.

This is also supported by the fact that curcumin constitutes $<5 \%(\mathrm{w} / \mathrm{w})$ of turmeric ${ }^{(15)}$, and other constituents might exhibit better activities without side effects ${ }^{(12)}$. Alternately, certain biological properties of turmeric are not caused by curcumin $^{(16)}$. It has been suggested that curcuminoid mixture possesses a superior therapeutic profile than curcumin alone for application in Alzheimer's disease ${ }^{(17)}$. These data suggest that the therapeutic properties of curcumin could be modulated in its natural milieu. Therefore, the neuroprotective effect of curcumin in the context of turmeric and the impact of other compounds should be examined to procure an improved therapeutic agent. Consequently, it is important to recapitulate the therapeutic properties of turmeric relevant to PD. Hence, our objective was to test turmeric as a neuroprotective agent in vivo.

Most studies have used acute administration of curcumin by systemic injections but not as dietary supplementation. In the present study, mice were chronically subjected to oral supplementation with turmeric and tested for its neuroprotective ability against the PD toxin 1-methyl-4-phenyl-1,2,3,6-tetrahydropyridine (MPTP).

\section{Experimental methods}

\section{Materials}

All chemicals and solvents used were of analytical grade. Fine chemicals and protease inhibitor cocktail were procured from Sigma (St Louis, MO, USA). PN was obtained from Upstate, Millipore (Billerica, MA, USA). Pure turmeric powder was a generous gift from Sadvaidyasala (Nanjanagud, Karnataka, India). Anti-tyrosine hydroxylase (TH) antibody was purchased from Santa Cruz Biotechnology, Inc. (Santa Cruz, CA, USA). Vectastain kit for immunohistochemistry was obtained from Vector Laboratories, Inc. (Burlingame, CA, USA).

\section{In vivo experiments}

All animal experiments were carried out in accordance with the institutional guidelines for the Care and Use of Laboratory Animals as per the internationally accepted principles for laboratory animal use and care. All experiments involving animals were approved by the institutional animal ethics committee. Adult male C57BL/6 mice (10-week-old, weight approximately $30 \mathrm{~g}$ each, six animals for each treatment) were obtained from the Central Animal Research Facility, NIMHANS, Bangalore, India. Mice were housed five per cage with access to a standard diet and water (or turmeric suspensions) ad libitum in a well-ventilated room, and all animals were exposed to a $12 \mathrm{~h}$ light $-12 \mathrm{~h}$ dark cycle.

In traditional Indian culinary methods, a small quantity of turmeric (approximately $5 \mathrm{mg} / \mathrm{d}$ per person) is added in daily cooking in aqueous media, and such dietary practice is followed throughout life. To simulate the oral consumption at higher concentrations, aqueous suspensions of turmeric ( 0.5 and $1 \%$ ) were prepared by boiling in water for $5 \mathrm{~min}$ and cooling. The animals had daily access to turmeric suspensions ad libitum as drinking-water for 3 months, simulating chronic dietary consumption. The presence of turmeric did not cause a significant change in the volume of water consumed by the animals. Furthermore, we observed that the consumption of turmeric did not cause any significant change in the body weight or activity of the animals (data not shown). In mice, the consumption of the turmeric suspension was approximately $10 \mathrm{ml} / \mathrm{d}$ per mouse corresponding to either $50 \mathrm{mg}$ (in $0.5 \%$ turmeric-fed mice) or $100 \mathrm{mg}$ (in $1 \%$ turmeric-fed mice) of turmeric powder. Consequently, the consumption of turmeric would be approximately $1.65 \mathrm{~g} / \mathrm{kg}$ body weight (in $0.5 \%$ turmeric-fed mice) or $3.3 \mathrm{~g} / \mathrm{kg}$ body weight (in $1 \%$ turmeric-fed mice) per $\mathrm{d}$ for 3 months. Although this mimics oral consumption of turmeric, the intake is significantly higher than that followed in humans.

Following turmeric consumption, animals were administered with a subcutaneous single injection of MPTP $(30 \mathrm{mg} / \mathrm{kg}$ body weight). Control animals were housed under identical conditions and received saline only by the same route. Animals were killed by decapitation at $24 \mathrm{~h}$ after the MPTP injection. Brains were recovered and utilised for either immunohistochemistry or biochemical experiments.

\section{Preparation of mitochondria and peroxynitrite treatment}

Preparation of brain mitochondria by the centrifugation method followed by PN treatment was carried out as previously described ${ }^{(5)}$. Briefly, PN solution was placed on the wall of the tube containing the mitochondria (suspended at $5 \mathrm{mg} / \mathrm{ml}$ protein concentration in $25 \mathrm{~mm}$-phosphate buffer $\mathrm{pH} 7 \cdot 2+5 \mathrm{~mm}^{-\mathrm{MgCl}_{2}}$ ) and vortex-mixed for a few seconds.

\section{Mitochondrial complex I enzyme assay}

Mitochondrial complex I (CI) enzyme assays were carried out as described earlier ${ }^{(5)}$. In brief, the assay was initiated by the addition of aliquots of brain mitochondria to $50 \mathrm{~mm}$-pottasium phosphate/Tris-HCl, pH 7.4, $500 \mu \mathrm{m}$-EDTA, $1 \%$ bovine serum albumin, $200 \mu \mathrm{m}-\mathrm{NADH}$ and $200 \mu \mathrm{m}$-decylubiquinone with and without $2 \mu \mathrm{m}$-rotenone in the presence of $\mathrm{KCN}$, with $0.002 \%$ dichloroindophenol as a secondary electron acceptor. The decrease in the absorbance at $600 \mathrm{~nm}$ was recorded as a measure of the enzyme reaction rate at $37^{\circ} \mathrm{C}$ for $10 \mathrm{~min}$, and 
specific activity was calculated. The results were plotted as relative rotenone-sensitive specific activity.

\section{Total glutathione (reduced + oxidised) estimation}

Total GSH in mouse brain extracts was estimated by the 5,5'-dithio-bis-2-nitro benzoic acid recycling method as described earlier ${ }^{(5,7)}$. Briefly, tissue/N27 cells were homogenised in phosphate EDTA (PE) buffer (100 mM-potassium phosphate buffer ( $\mathrm{pH} 7 \cdot 4$ ) containing 1 mM-EDTA), and total protein was estimated. The homogenate was precipitated with $2 \%$ sulfosalicylic acid (w/v), centrifuged (12000 rpm/ $15 \mathrm{~min}$ ), and the supernatant was used for the GSH assay. Then, $20 \mu \mathrm{l}$ of the supernatant was incubated with assay buffer (PE buffer containing 0.8 mM-5,5'-dithio-bis-2-nitro benzoic acid and $\mathrm{GSH}$ reductase $(0.32 \mathrm{U} / \mathrm{ml}))$ in a final reaction volume of $450 \mu \mathrm{l}$. The reaction was initiated by the addition of $0.6 \mathrm{~mm}-\mathrm{NADPH}$. The reaction kinetics of 5,5'-dithio-bis-2nitro benzoic acid recycling was monitored at $412 \mathrm{~nm}$ for $3 \mathrm{~min}$. The absolute GSH level in each sample was calculated based on oxidised GSH standards (0-250 ng) and normalised per mg protein.

\section{$\gamma$-Glutamyl cysteine ligase activity}

$\boldsymbol{\gamma}$-Glutamyl cysteine ligase ( $\boldsymbol{\gamma}$-GCL) enzyme activity was measured by the method described previously ${ }^{(18)}$. Briefly, brain samples were homogenised and sonicated in $1 \times \mathrm{PBS}$ and centrifuged at $9500 \mathrm{~g}$ for $10 \mathrm{~min}$ at $4^{\circ} \mathrm{C}$. The supernatant ( $40 \mu \mathrm{g}$ thick) was added to a reaction cocktail containing 100 mm-Tris-Cl (pH 8), $150 \mathrm{~mm}-\mathrm{KCl}, 5 \mathrm{~mm}-\mathrm{Na}_{2} \mathrm{ATP}, 2 \mathrm{~mm}-$ phosphoenol pyruvate, $10 \mathrm{~mm}$-L-glutamate, $20 \mathrm{mM}-\mathrm{MgCl}_{2}$, $2 \mathrm{~mm}-\mathrm{Na}_{2} \mathrm{EDTA}$ and pyruvate kinase/lactate dehydrogenase mix ( $17 \mathrm{U}$ each), and reaction kinetics was monitored at $340 \mathrm{~nm}$ for $10 \mathrm{~min}$. The reaction was initiated by the addition of $10 \mathrm{~mm}-\mathrm{L}-\alpha$-aminobutyrate. Assays run in the absence of $\mathrm{L}-\alpha$-aminobutyrate served as controls. Enzyme activity was normalised per mg protein.

\section{3-Nitrotyrosine Western blot}

To detect endogenous protein nitration, equal amounts of protein $(100 \mu \mathrm{g})$ from different brain and cell culture samples were spotted in triplicate onto a nitrocellulose membrane. The membrane was washed with PBS/Tween-20 followed by Western blot with a polyclonal anti-3-nitrotyrosine antibody. Band intensities in Western blots were quantified by a densitometric scanner, and the values were normalised against the respective anti- $\beta$-tubulin signal.

\section{Immunohistochemistry and stereology}

For immunohistochemistry, mice were perfused transcardially, first, with cold saline for $30 \mathrm{~min}$ followed by $4 \%$ paraformaldehyde (in $0 \cdot 1 \mathrm{M}$-sodium phosphate buffer, $\mathrm{pH} 7 \cdot 4$ ). The brains were then post-fixed in $4 \%$ paraformaldehyde for $48 \mathrm{~h}$. For stereological estimation, coronal sections $(40 \mu \mathrm{m})$ were taken through the entire SN (approximately -2.54 to -4.04 bregma) ${ }^{(19)}$ on a vibratome (Leica, Wetzlar, Germany), and every sixth section was processed for $\mathrm{TH}$ immunostaining. Briefly, free-floating sections were incubated in 1:1 sodium saline citrate buffer-formamide for $2 \mathrm{~h}$ at $65^{\circ} \mathrm{C}$, followed by washing in $2 \times$ sodium saline citrate buffer. Following this, endogenous peroxidase activity was quenched in 1:1 methanol-1 $\times$ PBS containing $3 \% \mathrm{H}_{2} \mathrm{O}_{2}$ for $30 \mathrm{~min}$ in dark. Sections were then blocked with normal horse serum (Vectastain Elite ABC kit; Vector Laboratories Inc., Burlingame, CA, USA) and incubated with a mouse monoclonal anti-TH primary antibody $(1: 100)$ for $72 \mathrm{~h}$ at $4{ }^{\circ} \mathrm{C}$ in a moist chamber. Sections were then incubated for $3 \mathrm{~h}$ in biotinylated universal secondary antibody followed by amplification with avidinbiotin complex (Vectastain Elite ABC kit) according to the manufacturer's instructions, and TH-positive cells were visualised using 3,3'-diamino benzidine tetrahydrochloride containing $0 \cdot 3 \% \mathrm{H}_{2} \mathrm{O}_{2}$.

Quantification of the TH-positive cells in the SN was performed using the optical fractionator method (Stereoinvestigator $^{\circledR}$; MBF Bioscience, Williston, VT, USA). Briefly, systemic random sampling sites with an unbiased counting frame $(100 \times 100 \mu \mathrm{m})$ were generated within the contour drawn around the SN. Counting of cells was done at $40 \times$ with an Olympus BX51 light microscope (Olympus, Tokyo, Japan), from every sixth section. Every cell that came into focus within the counting frame was counted across the serial sections, and final values were generated by Stereoinvestigator ${ }^{\circledR}$ software that was used for further analysis ${ }^{(20,21)}$.

\section{Statistical analyses}

All quantitative data were accumulated from at least three independent experiments. The final data are expressed as means with their standard errors. Differences between mean values were analysed by one-way ANOVA using Graphpad prism version 5.0 for windows software (Graphpad Software, Inc., San Diego, CA, USA). A $P$ value $<0.05$ was considered to be statistically significant in all experiments.

\section{Results}

In order to test the therapeutic application of turmeric, C57BL/6 mice were subjected to chronic dietary supplementation with 0.5 and $1 \%$ turmeric suspensions in drinkingwater for 3 months. Total brain GSH estimations in the whole-brain extracts showed a significant increase in mice fed with both 0.5 and $1 \%$ turmeric compared with untreated controls $(0.5 \%$ turmeric $=125 \%$ total GSH, $P<0.01 ; 1 \%$ turmeric $=130 \%, P<0.01$ compared with control $=100 \%$; Fig. 1(a)). However, frontal cortex, St and MB areas showed significantly increased GSH only in mice fed with $0.5 \%$ turmeric (frontal cortex $=122 \%$ total GSH, $P<0.001$; St $=144 \% ; \quad P<0.01 ; \quad \mathrm{MB}=128 \%, \quad P<0.01$ compared with control $=100 \%$ ) and not with $1 \%$ turmeric (Fig. 1(b)-(d)) Since GSH levels depend on the activity of the rate-limiting enzyme, $\gamma$-GCL, we tested whether turmeric caused an increase in GSH synthesis via induction of $\gamma$-GCL activity. We observed a significant increase in $\gamma$-GCL activity in the 
whole brain in both 0.5 and $1 \%$ turmeric-fed mice (approximately 2.5 -fold in $0.5 \%$ turmeric, $P<0.01$ and approximately 7.5-fold in $1 \%$ turmeric, $P<0.001$; Fig. $1(\mathrm{e})$ ). In the MB and St, similar to GSH levels, we observed an increase in $\gamma$-GCL activity only in $0.5 \%$ turmeric-fed mice (MB approximately
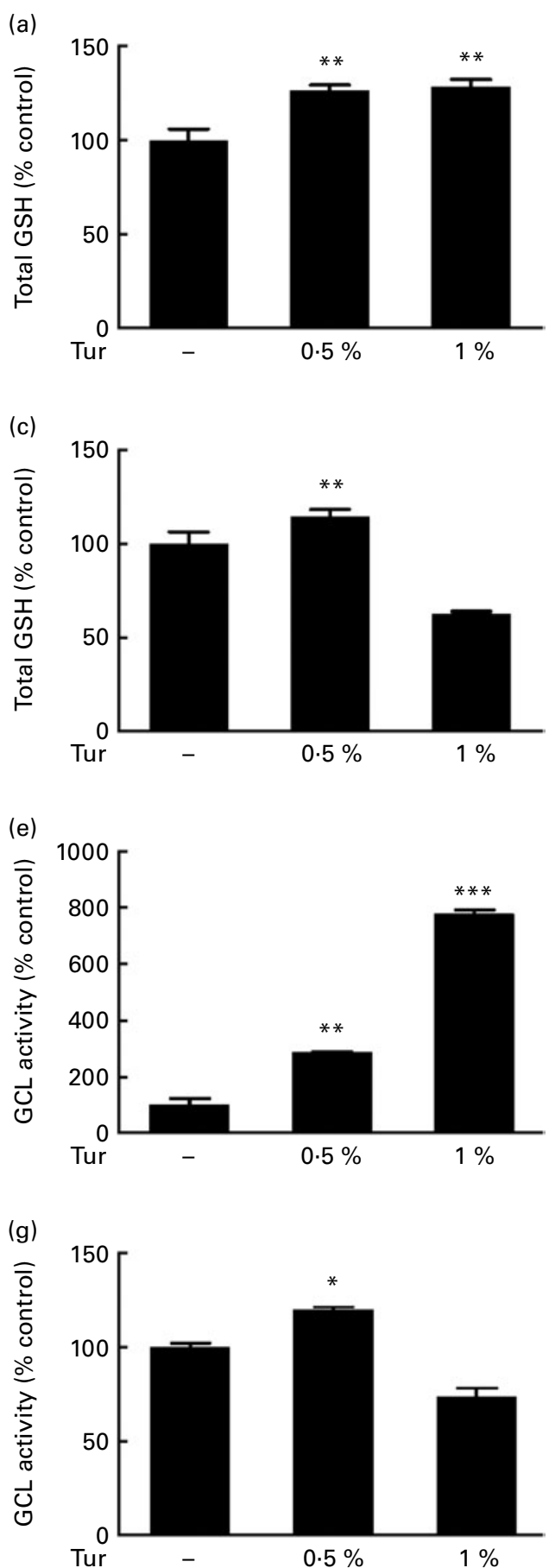

$80 \%, P<0.05$ and St approximately $20 \%, P<0.05)$ but not in $1 \%$ turmeric-fed mice (Fig. 1(f) and (g)). These data clearly suggest that the protective mechanism of turmeric oral supplementation is via an increase in $\gamma$-GCL activity, which in turn leads to an increase in GSH levels.
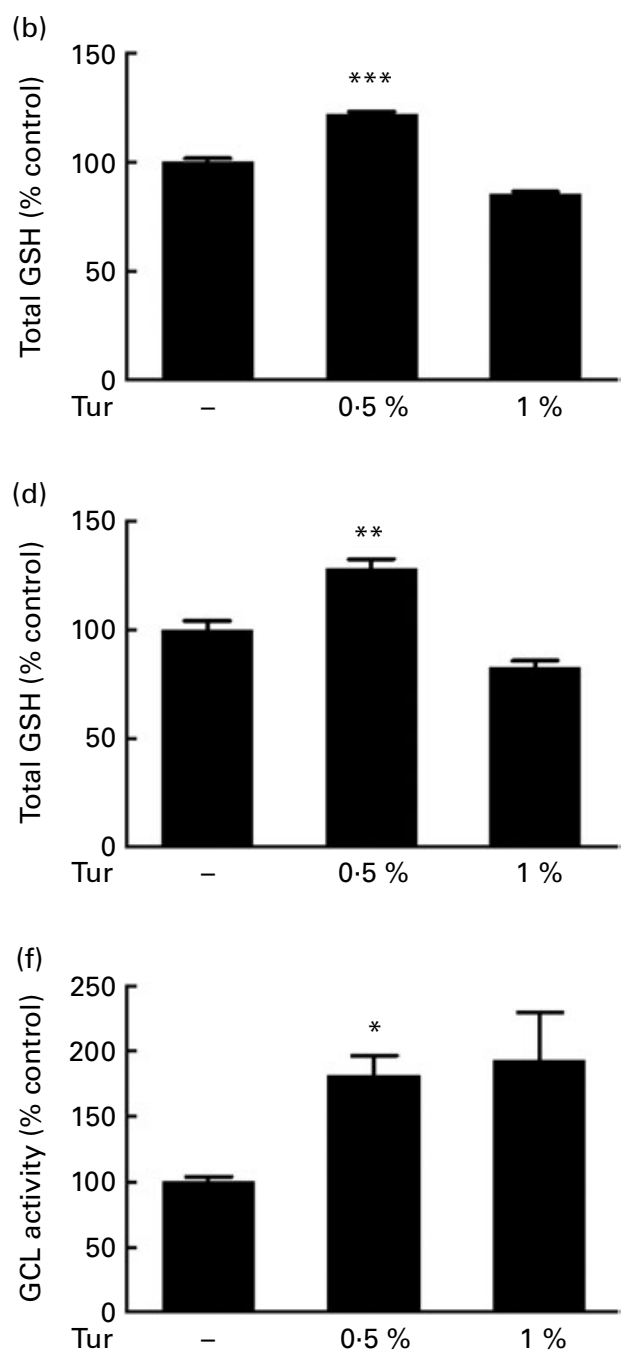

Fig. 1. Effect of dietary turmeric (Tur) on total glutathione (GSH) levels and $\gamma$-glutamyl cysteine ligase ( $\gamma$-GCL) activity in the mouse brain. Estimation of total brain GSH levels following 0.5 and $1 \%$ Tur diet in mice $(n 5)$ for 3 months. Values are percentage of GSH compared with untreated control $(100 \%$ total GSH $=1.6$ (SEM 0.15$) \mathrm{nmol} / \mathrm{mg}$ protein), with standard errors represented by vertical bars. Total GSH levels in the (a) whole brain, (b) frontal cortex, (c) striatum, and (d) midbrain are shown. Values were significantly different from those of control: ${ }^{\star *} P<0.01,{ }^{\star \star *} P<0.001$. Assay of $\gamma$-GCL activity in the same experimental animals following 0.5 and $1 \%$ Tur diet $(n 5)$ for 3 months is also shown as percentage of $\gamma$-GCL activity compared with untreated control (100\% $\gamma$-GCL activity $=583$ (SEM 199) $\mathrm{nmol} / \mathrm{min}$ per $\mathrm{mg}$ protein). Percentage activity in the (e) whole brain (f), midbrain and (g) striatum is shown. Values were significantly different from those of control: ${ }^{\star} P<0.05$, ${ }^{\star \star} P<0.01,{ }^{\star \star *} P<0.001$. 
We had previously demonstrated that an increase in brain GSH protected CI against nitrosative stress ${ }^{(5)}$. We observed that PN caused inhibition of CI activity in brain mitochondria (control $=100 \% \mathrm{CI}$ activity, $\mathrm{PN}=60 \%, P<0.001$ ), but this inhibition was prevented in the mitochondria from the whole brain $\quad(0.5 \%$ turmeric $+\mathrm{PN}=80 \% \quad \mathrm{CI}$ activity, $P<0.001 ; 1 \%$ turmeric $+\mathrm{PN}=80 \% \mathrm{CI}$ activity, $P<0.001 \mathrm{com}-$ pared with $\mathrm{PN}$ alone) in mice fed on a chronic turmeric diet (Fig. 2). The same trend was observed in different anatomical areas (frontal cortex: $0.5 \%$ turmeric $+\mathrm{PN}=80 \%, P<0.05 \%$; $1 \%$ turmeric $+\mathrm{PN}=90 \%, \quad P<0.001$ compared with $\mathrm{PN}$ alone; St: $\quad 0.5 \%$ turmeric $+\mathrm{PN}=80 \%, \quad P<0.001 \% ; \quad 1 \%$ turmeric $+\mathrm{PN}=74 \%, \quad P<0.05$ compared with $\mathrm{PN}$ alone; MB: $0.5 \%$ turmeric $+\mathrm{PN}=85 \%, P<0.001 \% ; 1 \%$ turmeric + $\mathrm{PN}=85 \%, P<0 \cdot 001$ compared with PN alone) (Fig. 2). These data indicate that turmeric improved the brain antioxidant load against mitochondrial damage with potential protection against PN toxicity.

Next, turmeric-fed mice were challenged with a single dose of MPTP (30 mg/kg body weight, subcutaneously). While MPTP administration inhibited CI activity in the MB $\quad(\mathrm{MPTP}=60 \%$ CI activity, $P<0.001$ compared with control $=100 \%$ ), turmeric consumption protected against the inhibition of CI activity (0.5\% turmeric + MPTP $=95 \%$, $P<0.001 ; 1 \%$ turmeric + MPTP $=140 \%, P<0.001$ compared with MPTP alone) (Fig. 3(a)). It has been established that MPTP-dependent mitochondrial damage is mediated partly via nitrosative stress ${ }^{(22)}$. Accordingly, quantification of total protein nitration by 3-nitrotyrosine dot blots showed that MPTP injections significantly increased protein nitration in
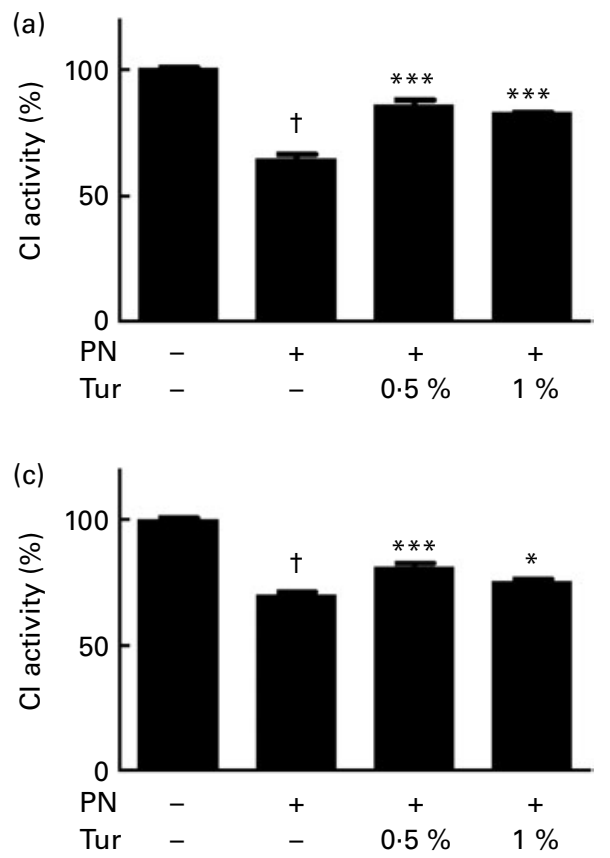

the MB mitochondria (MPTP $=150 \%$ 3-nitrotyrosine signal, $P<0.001$ compared with control $=100 \%$ ), indicating the role of nitrosative stress in mitochondrial damage and consequent neurodegeneration (Fig. 3(b)). Chronic turmeric consumption protected against CI inhibition via abolition of protein nitration $(0.5 \%$ turmeric $+\mathrm{MPTP}=112 \%, \quad P<0.05$; $1 \%$ turmeric + MPTP $=105 \%, P<0.01$ compared with MPTP alone) (Fig. 3). Total GSH estimations in turmeric-fed mice followed by MPTP injections showed that while MPTP alone caused a significant GSH depletion in the $\mathrm{MB}$ (control= $100 \%$, MPTP $=40 \%, P<0 \cdot 01)$, turmeric consumption caused a significant restoration of GSH levels ( $0.5 \%$ turmeric + $\mathrm{MPTP}=86 \%, \quad P<0.05 ; \quad 1 \% \quad$ turmeric $+\mathrm{MPTP}=112 \%$, $P<0.001$ compared with MPTP alone) (Fig. 4(a)). In the St, MPTP caused lesser GSH depletion (control $=100 \%$ $\mathrm{MPTP}=82 \%, P<0 \cdot 01)$, and turmeric consumption only at $1 \%$ caused significant restoration in GSH levels $(0.5 \%$ turmeric + MPTP $=80 \%$, NS; $1 \%$ turmeric + MPTP $=108 \%$, $P<0.01$ compared with MPTP) (Fig. 4(b)). It is therefore possible that turmeric could induce GSH synthesis and protect against nitrosative stress and mitochondrial damage with implications for the protection of SN dopaminergic neurons.

To prove this, whole brains from different experimental groups (MPTP only, turmeric only, turmeric + MPTP and control groups) were recovered, and the MB sections were subjected to anti-TH immunostaining and stereology-based quantification of TH-positive neurons. MPTP caused approximately $50 \%$ loss of TH-positive neurons $(P<0 \cdot 01)$, while exposure to turmeric alone did not cause significant toxicity


Fig. 2. Protective effect of dietary turmeric (Tur) on peroxynitrite (PN)-mediated mitochondrial complex I (Cl) inhibition. Quantification of $\mathrm{Cl}$ activity in brain mitochondria from mice $(n 5)$ fed on 0.5 and $1 \%$ Tur diet $( \pm 750 \mu \mathrm{M}-\mathrm{PN})$. Values are percentage of activity compared with $0 \mu \mathrm{M}-\mathrm{PN}(100 \% \mathrm{Cl}$ activity $=145(\mathrm{SEM}$ 3.5) $\mathrm{nmol} / \mathrm{min}$ per $\mathrm{mg}$ of mitochondrial protein), with standard errors represented by vertical bars. Cl activities in the (a) whole brain, (b) frontal cortex, (c) striatum, and (d) midbrain are shown. Values were significantly different from those of PN treated (in Tur-fed mice; ${ }^{\star} P<0.05,{ }^{\star \star \star} P<0.001$ ) and control (in $P N$ treated; † $P<0.001$ ). 

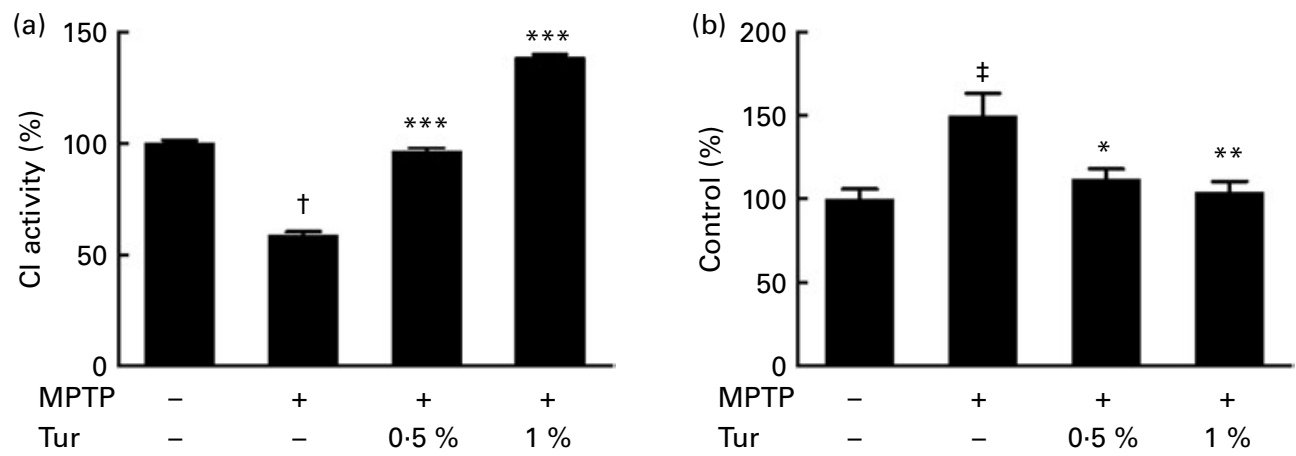

Fig. 3. Effect of dietary turmeric (Tur) on mitochondrial complex I (Cl) activity and protein nitration in the midbrain of 1-methyl-4-phenyl-1,2,3,6-tetrahydropyridine (MPTP)-injected animals. (a) $\mathrm{Cl}$ activity in brain mitochondria from mice $(n 5)$ fed on 0.5 and $1 \%$ Tur diet + MPTP injection ( $30 \mathrm{mg} / \mathrm{kg}$ body weight, single subcutaneous injection). Values were significantly different from those of MPTP only (in Tur-fed mice): ${ }^{* * *} P<0.001$ and values were significantly different from those of control (in MPTP only): $† P<0.001$. (b) $\alpha$-3-Nitrotyrosine Western blot analysis represents the relative abundance of protein nitration in brain mitochondria as in (a) ( $n$ 5). Values were significantly different from those of MPTP only (in Tur-fed mice; ${ }^{*} P<0.05,{ }^{* *} P<0.01$ ) and control (in MPTP only; $\ddagger P<0.01$ ).

to the dopaminergic neurons (Fig. 5). Mice chronically fed with turmeric showed protection against MPTP and displayed approximately $30 \%$ more neurons in both the $0.5 \%$ $(P<0.05)$ and $1 \%(P<0.05)$ turmeric-fed mice compared with MPTP-treated mice. Therefore, dietary supplementation with turmeric protects against MPTP-mediated degeneration of dopaminergic neurons (Fig. 5) probably via a GSHdependent mechanism.
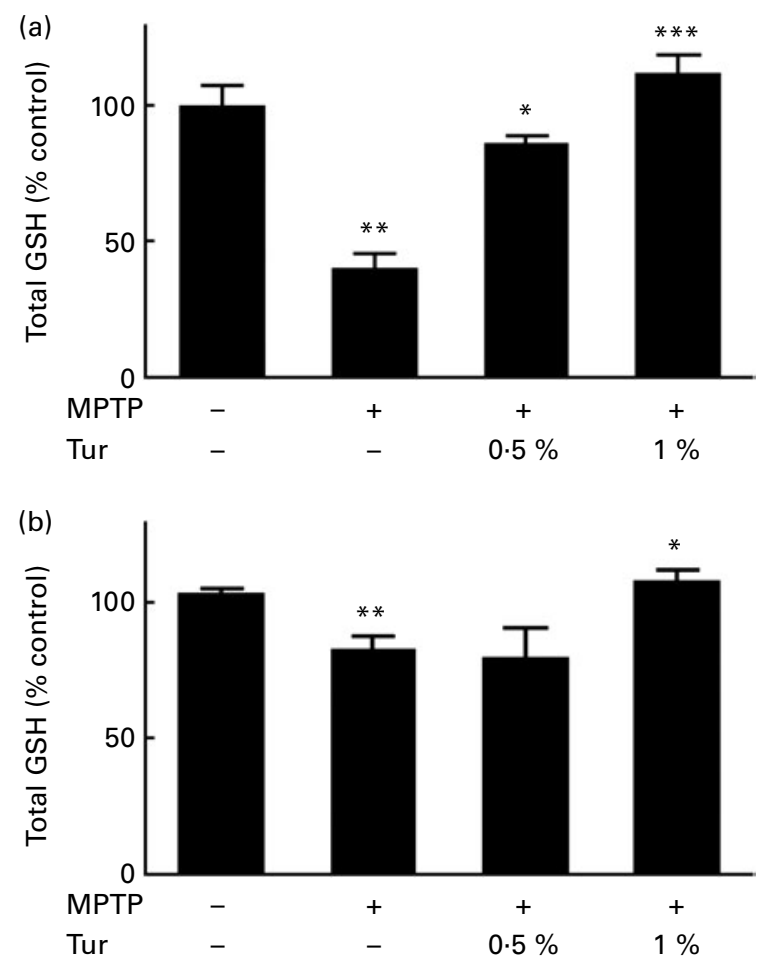

Fig. 4. Effect of dietary turmeric (Tur) on brain glutathione (GSH) levels in mice treated with 1-methyl-4-phenyl-1,2,3,6-tetrahydropyridine (MPTP). Estimation of total GSH levels in the brain of mice fed on 0.5 and $1 \%$ Tur diet + MPTP injection ( $30 \mathrm{mg} / \mathrm{kg}$ body weight, single subcutaneous injection). Values are percentage of GSH compared with untreated control, with standard errors represented by vertical bars. Total GSH levels in the (a) midbrain and (b) striatum are shown. Values were significantly different both in MPTP only (compared with control) and in Tur-fed mice (compared with MPTP only): ${ }^{*} P<0.05,{ }^{* *} P<0.01$ and ${ }^{\star \star *} P<0.001$.

\section{Discussion}

The inherent complexity in the pathogenesis of PD has impeded the screening for potential PD drugs. It has been established that the interplay among genetic, dietary and environmental factors determines the occurrence of the disease $^{(23-25)}$. Epidemiological studies have indicated that the incidence of PD is low in India ${ }^{(26)}$. On the other hand, the prevalence of PD is higher in Caucasian than in non-Caucasian communities, and it increases with advancing age, indicating that race and age might play a role in the pathogenesis of PD. A study exploring this difference found that compared with Caucasian human brains, Indian brains showed no loss of melanised nigral neurons with advancing age ${ }^{(27)}$. Furthermore, the absolute number of these melanised neurons was approximately $40 \%$ lower in Indian brains than in Caucasian brains. Since there were no differences in dopamine content and motor function, it could be surmised that it is not the absolute number of nigral neurons but their percentage loss that contribute to dopamine deficiency in PD. Lower prevalence of PD among Indians, despite having fewer nigral neurons, indicates undefined protective mechanisms that prevent the loss of nigral neurons with age ${ }^{(27)}$. In a related study, it has been reported that there was no age-related loss of nigral function in Asian Indians unlike in Americans, which could explain the lower incidence of PD in Asian Indians ${ }^{(28)}$. A similar study in Anglo-Indians, an admixed population of European and Indian origin, has concluded that instead of displaying an average of European and Indian prevalence, the Anglo-Indians showed reduced occurrence of $\mathrm{PD}^{(29)}$. These studies have indicated that genetic and dietary factors might play an important role in reducing the occurrence of PD among the Asian Indians.

Indian diet and traditional cuisine involves the use of several spices as digestive stimulants ${ }^{(30)}$. Apart from immediate beneficial effects, chronic consumption of spices such as turmeric is suggested to provide antioxidant defence and protection against diseases such as diabetes ${ }^{(31)}$. Studies of Indian immigrants in Western countries indicated increased rates of chronic diseases such as CHD and cancer, which could be attributed to a change in dietary practices ${ }^{(32)}$. Analysis of the 
(a)

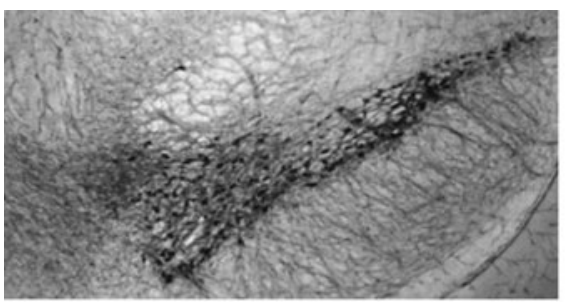

(c)

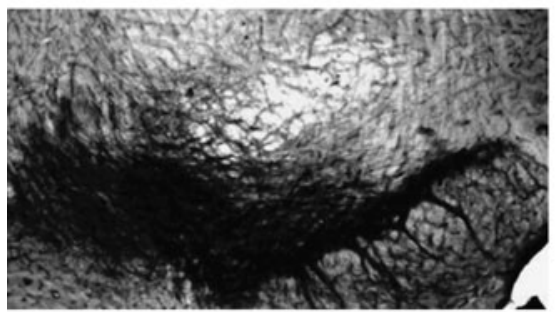

(e)

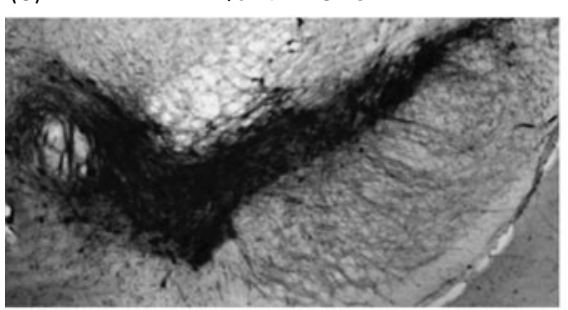

(b)

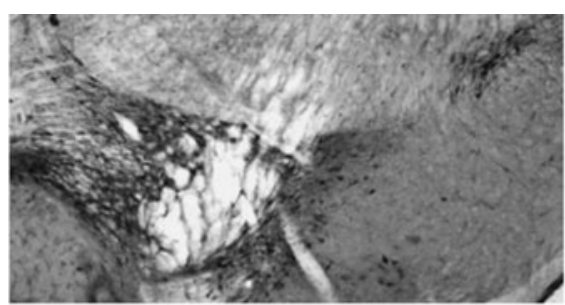

(d)

$0.5 \%$ Turmeric + MPTP

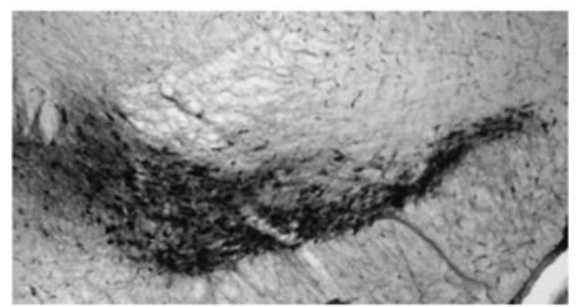

(f)

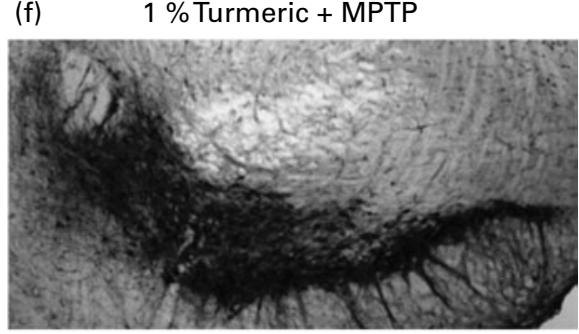

(g)

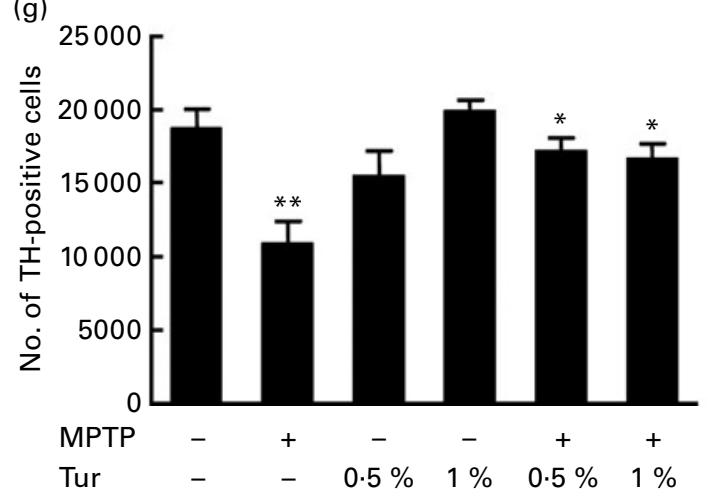

Fig. 5. Dietary turmeric (Tur) protects against 1-methyl-4-phenyl-1,2,3,6-tetrahydropyridine (MPTP)-mediated dopaminergic neuronal cell death. Representative sections of the substantia nigra (SN) showing tyrosine hydroxylase (TH) immunostaining in control (a), MPTP alone ( $30 \mathrm{mg} / \mathrm{kg}$ body weight, single subcutaneous injection) (b), Tur fed $(0.5$ and $1 \%$ orally for 3 months) ((c) and (e), respectively) and Tur fed $(0.5$ and $1 \%)+$ MPTP-injected mice ((d) and (f), respectively). (g) Stereology-based quantification of TH-positive neurons in the $\mathrm{SN}$ in groups as in (a). Values were significantly different from those of controls $\left({ }^{* *} P<0.01\right)$ and MPTP treated $\left({ }^{*} P<0.05\right)$. Three animals or more in each group.

cancer risk in India indicated lower cancer rates compared with Western countries. But these rates could rise with increasing population migration, and changes in lifestyles and diet. Dietary practices in India based on centuries-old cultural and religious principles, which focus on vegetarian foods and use of spices, might play a role in cancer prevention ${ }^{(32)}$. The role of common Indian spices such as turmeric in cancer prevention has been well studied ${ }^{(33)}$. These studies have highlighted the role of Indian dietary spices with special emphasis on chronic consumption of turmeric in disease prevention. However, it has to be understood whether chronic consumption of turmeric in diet throughout life could be more beneficial and have a neuroprotective effect in the brain.

To test the neuroprotective effect of chronic turmeric consumption, we fed mice with aqueous suspensions of turmeric for 3 months. We observed that dietary turmeric could protect SN neurons from MPTP-mediated neurotoxicity (Fig. 5), mitochondrial damage and protein nitration (Fig. 3). While total GSH levels increased with 0.5 and $1 \%$ turmeric in the whole brain, the trends were different in different regions (Fig. 1). Although the MB showed decreased GSH in $1 \%$ turmericfed mice compared with $0.5 \%$, the levels were similar to 
that found in controls. Whereas in the frontal cortex and St, the $1 \%$ turmeric diet caused a significant decrease in total GSH levels compared with $0.5 \%$ turmeric and control groups. It is possible that different molecular species in the turmeric suspension could target GSH metabolism and related pathways by yet undefined mechanisms. Also, there might be a region-specific response to turmeric in the brain. However, such discrepancy did not have a significant bearing on the survival of the dopaminergic neurons. Mice fed on $1 \%$ turmeric alone did not show a significant change in the number of TH-positive neurons in the SN compared with controls, suggesting that turmeric concentrations in this range are non-toxic (Fig. 4). In few mice on a turmeric diet, a significant increase in TH-positive neurons compared with untreated controls was observed (data not shown). This is consistent with previous reports ${ }^{(34,35)}$, suggesting that the potential neuroprotective effect of turmeric is mediated via enhanced neurogenesis.

It has been established that MPTP caused mitochondrial damage and consequent neurodegeneration partly by a nitric oxide synthase-mediated increase in protein nitration ${ }^{(22)}$. Consistent with this, we observed that turmeric could prevent MPTP toxicity in vivo, which was partly mediated by increasing GSH content and preventing protein nitration (Figs. 3 and 4). We suggest that turmeric extract provides a complicated mechanism of neuromodulatory effects involving multiple pathways, which need to be addressed in detail. There have been previous studies that utilised turmeric extracts as antioxidant and protective agents. The aqueous extracted turmeric antioxidant protein protected against $\mathrm{H}_{2} \mathrm{O}_{2}$-induced erythrocyte lipid peroxidation and haemolysis, and has been found to be more effective as an antioxidant than tocopherol and curcumin $^{(36)}$. Similarly, Sarhan et al. ${ }^{(37)}$ studied the protective effect of turmeric, Ginkgo biloba, silymarin separately or in combination, on Fe-dependent oxidative stress and lipid peroxidation in rats. They reported that pre-treatment with turmeric significantly induced blood lysate GSH levels and a significant rise in hepatic superoxide dismutase.

In the field of therapeutics and nutraceuticals, there is a constant disparity between scientists utilising pure compounds and those using natural extracts. Consequently, the challenge has been to identify compound(s) in turmeric that contribute to its neuroprotective function. The most relevant experiment in this direction would be to prepare aqueous and ethanolic extracts of turmeric followed by thorough fractionation and comprehensive testing in experimental models of PD. Such compound(s) must have antioxidant, anti-inflammatory and neuroprotective features at a particular dosage.

According to the literature, curcumin has emerged as the most active ingredient of turmeric with implications for neuroprotection. The neuroprotective property of curcumin could be mediated by its antioxidant property ${ }^{(5-8,38)}$, regulation of gene expression ${ }^{(4)}$, protection of mitochondria $^{(39)}$, prevention of protein aggregation ${ }^{(40)}$, protection against dopamine neurotoxicity, microglial activation and neuroinflammation $^{(41)}$ and regulation of cellular stress response ${ }^{(42)}$. Consequently, curcumin has been neuroprotective in different models of $\mathrm{PD}^{(38,43-48)}$. Although it is tempting to assign the neuroprotective property of turmeric to curcumin due to an overwhelming support from the literature, we do not have direct evidence in the present study to show that the neuroprotective ability of turmeric is mediated via curcumin. Survey of plant extract-based studies clearly indicates that the biological property of an extract need not be dependent on a single compound. It should also be noted that even though curcumin is the major polyphenol in turmeric, it constitutes $<5 \%(\mathrm{w} / \mathrm{w})$ of the spice ${ }^{(15)}$, suggesting that other components of turmeric might be involved. Several compounds with potential medicinal properties have been isolated from turmeric and characterised. These include novel curcuminoids, sesquiterpenes, calebin derivatives and related compounds ${ }^{(49-52)}$. Such compounds have been tested in experimental models of neurodegeneration ${ }^{(51-53)}$. Furthermore, the utilisation of isolated compounds such as curcumin suffers a major disadvantage due to poor bioavailability ${ }^{(10)}$. Schiborr et al. ${ }^{(54)}$ reported that in the brains of mice forcefed $(50 \mathrm{mg} / \mathrm{kg}$ body weight) or intraperitoneally injected (100 mg/kg body weight) with curcumin, its levels were below the limit of detection at $0 \cdot 5,1$ and $2 \mathrm{~h}$ after oral administration and reached only $4-5 \mu \mathrm{g} / \mathrm{g}$ brain $20-40 \mathrm{~min}$ after the intraperitoneal injection. Similarly, Suresh \& Srinivasan ${ }^{(55)}$ reported that following an oral administration of curcumin ( $500 \mathrm{mg} / \mathrm{kg}$ body weight), its concentration was maximum in the intestine at $1 \mathrm{~h}$, maximum in the blood at $6 \mathrm{~h}$ and remained at significantly higher level even at $24 \mathrm{~h}$. The bioavailability of curcumin in body tissues including the brain was improved only when administered concomitantly with piperine.

Therefore, it would be interesting to extend such studies regarding the pharmacodynamics and pharmacokinetics to other constituents of turmeric in the brain. This would definitely help in analysing the bioavailability and biological activity of turmeric components and ascertain how they are modulated in their natural milieu by other compounds. This would also help us to assign all the neuroprotective compounds in turmeric and would support the use of turmeric instead of isolated compounds, for PD therapy.

\section{Conclusions}

Our data indicate that chronic exposure to turmeric through diet could increase the brain antioxidant load and protect against oxidative and nitrosative stress, ultimately preventing neurodegeneration. However, our data also emphasise the re-evaluation of turmeric and further characterise its components that could modulate neuronal viability and protect against neurotoxicity. This could be used in the development of improved dietary practices and therapies with fewer adverse effects in vivo.

\section{Acknowledgements}

The present study was supported by a fast-track grant from DST, India (to M. M. S. B.). R. B. M. and J. V. gratefully acknowledge the receipt of Senior Research Fellowship from Council for Scientific and Industrial Research, India. G. H. gratefully acknowledges the receipt of Junior Research 
Fellowship from the Indian Council for Medical Research, India. M. M. S. B. and R. B. M. designed the study. R. B. M., J. V. and G. H. conducted the experiments. M. M. S. B., B. S. S. R. and R. B. M. analysed the data. M. M. S. B. and R. B. M. wrote the manuscript. The authors state that there are no conflicts of interest.

\section{References}

1. Burke RE (1998) Parkinson's disease. In Cell Death and Disease of the Nervous System, pp. 459-475 [VE Koliatsos and RR Ratan, editors]. Totowa, NJ: Humana.

2. Bharath MM (2008) Therapeutic strategies in Parkinson's disease. Recent Patents on Endocrine, Metabolic \& Immune Drug Discovery 2, 135-147.

3. Aggarwal BB, Bhatt ID, Ichikawa H, et al. (2006) Curcumin biological and medicinal properties. In Turmeric: The Genus Curcuma, pp. 297-368. London: Taylor and Francis Group.

4. Goel A, Kunnumakkara AB \& Aggarwal BB (2008) Curcumin as 'Curecumin': from kitchen to clinic. Biochem Pharmacol 75, 787-809.

5. Mythri RB, Jagatha B, Pradhan N, et al. (2007) Mitochondrial complex I inhibition in Parkinson's disease: how can curcumin protect mitochondria? Antioxid Redox Signal 9, 399-408.

6. Harish G, Venkateshappa C, Mythri RB, et al. (2010) Bioconjugates of curcumin display improved protection against glutathione depletion mediated oxidative stress in a dopaminergic neuronal cell line: implications for Parkinson's disease. Bioorg Med Chem 18, 2631-2638.

7. Jagatha B, Mythri RB, Vali S, et al. (2008) Curcumin treatment alleviates the effects of glutathione depletion in vitro and in vivo: therapeutic implications for Parkinson's disease explained via in silico studies. Free Radic Biol Med $\mathbf{4 4}$, 907-917.

8. Ramassamy C (2006) Emerging role of polyphenolic compounds in the treatment of neurodegenerative diseases: a review of their intracellular targets. Eur J Pharmacol 545 , 51-64.

9. Ammon HP \& Wahl MA (1991) Pharmacology of Curcuma longa. Planta Med 57, 1-7.

10. Anand P, Kunnumakkara AB, Newman RA, et al. (2007) Bioavailability of curcumin: problems and promises. Mol Pharm 4, 807-818.

11. Sharma RA, Steward WP \& Gescher AJ (2007) Pharmacokinetics and pharmacodynamics of curcumin. Adv Exp Med Biol 595, 453-470.

12. Balaji S \& Chempakam B (2009) Pharmacokinetics prediction and drugability assessment of diphenylheptanoids from turmeric (Curcuma longa L.). Med Chem 5, 130-138.

13. Ortiz-Ortiz MA, Moran JM, Ruiz-Mesa LM, et al. (2010) Curcumin exposure induces expression of the Parkinson's disease-associated leucine-rich repeat kinase 2 (LRRK2) in rat mesencephalic cells. Neurosci Lett 468, 120-124.

14. Ortiz-Ortiz MA, Moran JM, Bravosanpedro JM, et al. (2009) Curcumin enhances paraquat-induced apoptosis of N27 mesencephalic cells via the generation of reactive oxygen species. Neurotoxicology 30, 1008-1018.

15. Tayyem RF, Heath DD, Al-Delaimy WK, et al. (2006) Curcumin content of turmeric and curry powders. Nutr Cancer $\mathbf{5 5}$, $126-131$.

16. Hou XL, Takahashi K, Kinoshita N, et al. (2007) Possible inhibitory mechanism of Curcuma drugs on CYP3A4 in 1alpha,25 dihydroxyvitamin $\mathrm{D}_{3}$ treated Caco-2 cells. Int $J$ Pharm 337, 169-177.
17. Ahmed T \& Gilani AH (2009) Inhibitory effect of curcuminoids on acetylcholinesterase activity and attenuation of scopolamine-induced amnesia may explain medicinal use of turmeric in Alzheimer's disease. Pharmacol Biochem Behav 91, 554-559.

18. Seelig GF \& Meister A (1985) Glutathione biosynthesis; gamma-glutamylcysteine synthetase from rat kidney. Methods Enzymol 113, 379-390.

19. Paxinos G \& Franklin KBJ (2001) The Mouse Brain in Stereotaxic Co-ordinates, 2nd ed. San Diego, CA: Academic Press.

20. Veena J, Srikumar BN, Mahati K, et al. (2009) Enriched environment restores hippocampal cell proliferation and ameliorates cognitive deficits in chronically stressed rats. $J$ Neurosci Res $\mathbf{8 7}, 831-843$.

21. Veena J, Srikumar BN, Raju TR, et al. (2009) Exposure to enriched environment restores the survival and differentiation of new born cells in the hippocampus and ameliorates depressive symptoms in chronically stressed rats. Neurosci Lett 455, 178-182.

22. Ebadi M \& Sharma SK (2003) Peroxynitrite and mitochondrial dysfunction in the pathogenesis of Parkinson's disease. Antioxid Redox Signal 5, 319-335.

23. Abeliovich A \& Flint Beal M (2006) Parkinsonism genes: culprits and clues. J Neurochem 99, 1062-1072.

24. Cicchetti F, Drouin-Ouellet J \& Gross RE (2009) Environmental toxins and Parkinson's disease: what have we learned from pesticide-induced animal models? Trends Pharmacol Sci 30, 475-483.

25. Mattson MP (2003) Gene-diet interactions in brain aging and neurodegenerative disorders. Ann Intern Med 139, 441-444.

26. Bharucha NE, Bharucha EP, Bharucha AE, et al. (1988) Prevalence of Parkinson's disease in the Parsi community of Bombay, India. Arch Neurol 45, 1321-1323.

27. Muthane U, Yasha TC \& Shankar SK (1998) Low numbers and no loss of melanized nigral neurons with increasing age in normal human brains from India. Ann Neurol 43, 283-287.

28. Alladi PA, Mahadevan A, Yasha TC, et al. (2009) Absence of age-related changes in nigral dopaminergic neurons of Asian Indians: relevance to lower incidence of Parkinson's disease. Neuroscience 159, 236-245.

29. Ragothaman M, Murgod UA, Gururaj G, et al. (2003) Lower risk of Parkinson's disease in an admixed population of European and Indian origins. Mov Disord 18, 912-914.

30. Platel K \& Srinivasan K (2004) Digestive stimulant action of spices: a myth or reality? Indian J Med Res 119, 167-179.

31. Srinivasan K (2005) Plant foods in the management of diabetes mellitus: spices as beneficial antidiabetic food adjuncts. Int J Food Sci Nutr 56, 399-414.

32. Sinha R, Anderson DE, McDonald SS, et al. (2003) Cancer risk and diet in India. J Postgrad Med 49, 222-228.

33. Anand P, Sundaram C, Jhurani S, et al. (2008) Curcumin and cancer: an 'old-age' disease with an 'age-old' solution. Cancer Lett 267, 133-164.

34. Kim SJ, Son TG, Park HR, et al. (2008) Curcumin stimulates proliferation of embryonic neural progenitor cells and neurogenesis in the adult hippocampus. J Biol Chem 283, 14497-14505.

35. Xu Y, Ku B, Cui L, et al. (2007) Curcumin reverses impaired hippocampal neurogenesis and increases serotonin receptor $1 \mathrm{~A}$ mRNA and brain-derived neurotrophic factor expression in chronically stressed rats. Brain Res 1162, 9-18.

36. Lalitha S \& Selvam R (1999) Prevention of $\mathrm{H}_{2} \mathrm{O}_{2}$-induced red blood cell lipid peroxidation and hemolysis by aqueous extracted turmeric. Asia Pac J Clin Nutr 8, 113-114. 
37. Sarhan R, El-Azim SA, Motawi TM, et al. (2007) Protective effect of turmeric, Ginkgo biloba, Silymarin separately or in combination, on iron-induced oxidative stress and lipid peroxidation in rats. Int J Pharmacol 3, 375-384.

38. Wang J, Du XX, Jiang H, et al. (2009) Curcumin attenuates 6-hydroxydopamine-induced cytotoxicity by anti-oxidation and nuclear factor-kappa B modulation in MES23.5 cells. Biochem Pharmacol 78, 178-183.

39. Beal MF (2009) Therapeutic approaches to mitochondrial dysfunction in Parkinson's disease. Parkinsonism Relat Disord 15, Suppl. 3, S189-S194.

40. Pal R, Cristan EA, Schnittker K, et al. (2010) Rescue of ER oxidoreductase function through polyphenolic phytochemical intervention: implications for subcellular traffic and neurodegenerative disorders. Biochem Biophys Res Commun 392, 567-571.

41. Yang S, Zhang D, Yang Z, et al. (2008) Curcumin protects dopaminergic neuron against LPS induced neurotoxicity in primary rat neuron/glia culture. Neurochem Res $\mathbf{3 3}$ 2044-2053.

42. Calabrese V, Guagliano E, Sapienza M, et al. (2006) Redox regulation of cellular stress response in neurodegenerative disorders. Ital J Biochem 55, 263-282.

43. Sawada H, Ibi M, Kihara T, et al. (2002) Estradiol protects dopaminergic neurons in a MPP + Parkinson's disease model. Neuropharmacology 42, 1056-1064.

44. Yu S, Zheng W, Xin N, et al. (2010) Curcumin prevents dopaminergic neuronal death through inhibition of the c-Jun N-terminal kinase pathway. Rejuvenation Res 13, 55-64.

45. Rajeswari A \& Sabesan M (2008) Inhibition of monoamine oxidase- $\mathrm{B}$ by the polyphenolic compound, curcumin and its metabolite tetrahydrocurcumin, in a model of Parkinson's disease induced by MPTP neurodegeneration in mice. Inflammopharmacology 16, 96-99.

46. Chen J, Tang XQ, Zhi JL, et al. (2006) Curcumin protects PC12 cells against 1-methyl-4-phenylpyridinium ion-induced apoptosis by bcl-2-mitochondria-ROS-iNOS pathway. Apoptosis 11, 943-953.

47. Zbarsky V, Datla KP, Parkar S, et al. (2005) Neuroprotective properties of the natural phenolic antioxidants curcumin and naringenin but not quercetin and fisetin in a 6-OHDA model of Parkinson's disease. Free Radic Res 39, 1119-1125.

48. Pandey N, Strider J, Nolan WC, et al. (2008) Curcumin inhibits aggregation of alpha-synuclein. Acta Neuropathol 115, 479-489.

49. Li W, Wang S, Feng J, et al. (2009) Structure elucidation and NMR assignments for curcuminoids from the rhizomes of Curcuma longa. Magn Reson Chem 47, 902-908.

50. Zeng Y, Qiu F, Takahashi K, et al. (2007) New sesquiterpenes and calebin derivatives from Curcuma longa. Chem Pharm Bull (Tokyo) 55, 940-943.

51. Kim DS, Park SY \& Kim JK (2001) Curcuminoids from Curcuma longa L. (Zingiberaceae) that protect PC12 rat pheochromocytoma and normal human umbilical vein endothelial cells from betaA(1-42) insult. Neurosci Lett 303, 57-61.

52. Kim DS \& Kim JY (2001) Total synthesis of Calebin-A, preparation of its analogues, and their neuronal cell protectivity against beta-amyloid insult. Bioorg Med Chem Lett 11, 2541-2543.

53. Park SY \& Kim DS (2002) Discovery of natural products from Curcuma longa that protect cells from beta-amyloid insult: a drug discovery effort against Alzheimer's disease. J Nat Prod 65, 1227-1231

54. Schiborr C, Eckert GP, Rimbach G, et al. (2010) A validated method for the quantification of curcumin in plasma and brain tissue by fast narrow-bore high-performance liquid chromatography with fluorescence detection. Anal Bioanal Chem 397, 1917-1925.

55. Suresh D \& Srinivasan K (2010) Tissue distribution \& elimination of capsaicin, piperine \& curcumin following oral intake in rats. Indian J Med Res 131, 682-691. 